\title{
ANTIOXIDANT NUTRIENTS IN PREGNANCY: A SYSTEMATIC REVIEW OF THE LITERATURE
}

\section{FIONA MATHEWS}

Department of Public Health and Primary Care, Radcliffe Infirmary, University of Oxford, OX2 6HE, U.K.

\section{CONTENTS}

\section{INTRODUCTION}

SCOPE OF REVIEW

IDENTIFICATION OF PAPERS

REVIEW CRITERIA

Birth weight and/or intra-uterine growth retardation

Zinc supplement trials

Conclusions from the supplement trials

Cohort studies

Case-control studies

Conclusions from the cohort and case-control studies

Preterm delivery and/or gestational age at delivery

Conclusions

SUMMARY OF THE EFFECT OF ZINC ON PREGNANCY OUTCOME

VITAMIN C AND PREGNANCY OUTCOME

BACKGROUND

RESULTS

Mean birth weight, IUGR and low birth weight

Preterm delivery and/or gestational age at delivery

CAROTENOIDS AND PREGNANCY OUTCOME 


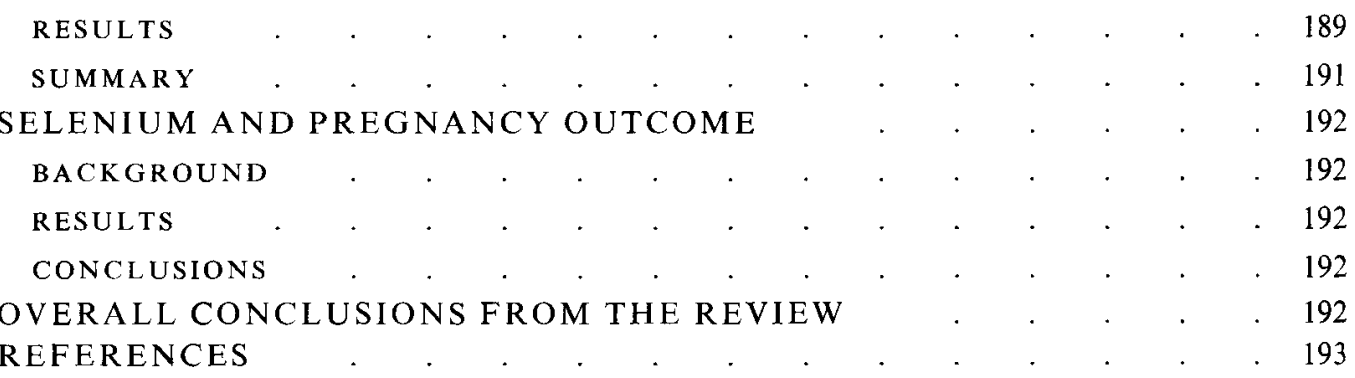

\section{INTRODUCTION}

In 1987 Kramer published a systematic literature review (Kramer, 1987) appraising studies published prior to 1984 which analysed the effects of maternal nutrition on fetal growth and development. Since then large amounts of new data have become available, and there has also been increased interest in nutritional issues among both health professionals and the lay public. The conclusive evidence, published in 1991, that folate supplements are effective in preventing neural tube defects in women at risk (MRC Vitamin Study Research Group, 1991) has bolstered interest in the use of nutrient supplements during pregnancy. Many pregnant women now take folic acid supplements (most of which also contain other nutrients), and the use of other types of nutritional supplement during pregnancy is also very common.

A search of the literature reveals that the evidence for providing supplements or dietary advice (other than in relation to folate) appears flimsy. Nevertheless, supplements continue to be taken, no doubt encouraged by the popular belief that nutrient supplements, even if not therapeutic, must be benign since their constituents occur naturally in food. However, careful scrutiny must be given to the scientific evidence for the use of such interventions. The potential dangers have already been demonstrated in humans: programmes of high dose vitamin A supplementation in Africa have resulted in embryonic teratogenesis, yet levels of retinol intake well above recommended doses have been seen in the UK among pregnant women taking high dose cod liver oil supplements in addition to multivitamins (F. Mathews \& A. W. Neil, unpublished). There is also now evidence that routine iron supplementation in non-deficient pregnant women can increase rates of preterm delivery and low birth weight (Mahomed, 1993c). Further, the possibility exists that supplementation with one nutrient can adversely affect the absorption and metabolism of others. For example, work on animals has demonstrated that fetal malformations are $45 \%$ higher when zinc deficient animals are given calcium supplements than when the animals are deficient in both nutrients (Hurley \& Tao, 1972). A systematic review of the literature is therefore necessary to ascertain whether there are grounds to perform randomized controlled trials to test the effects of nutrition on mean birth weight, mean gestational age, intrauterine growth retardation (IUGR) ${ }^{1}$ and preterm birth. Only literature published after 1983 has been included in the review because most earlier literature has already been well summarized in Kramer's paper (Kramer, 1987).

This review focuses on the role of micro- rather than macro-nutrients during pregnancy. Rush (1989) has already conducted a systematic review of studies which investigated protein and energy intake in pregnancy. He concluded that well intentioned programmes of dietary supplementation with these nutrients have not always had the desired effects:

] Intrauterine growth retardation (IUGR), and small for gestational age (SGA) will be used interchangeably in this text. 
high density protein supplements were consistently associated with a depression of mean birth weight. Balanced supplements of protein and energy did seem to produce increases in birth weight, but these increments were small, averaging only $30-50 \mathrm{~g}$.

While it is known that sudden and severe restriction of energy intake during pregnancy (such as in the Dutch Hunger Winter) can reduce birth weight by up to $300 \mathrm{~g}$ and increase the incidence of preterm delivery and other adverse outcomes (Antonov, 1947, Stein et al. 1975), the relationship between energy intake and pregnancy outcomes under other conditions remains unclear. Similarly, the individual effects of carbohydrate, fat, and protein intakes on pregnancy outcome are unresolved. Supplementation studies have produced both increases and decreases in mean birth weight (differences ranging from 32 to $114 \mathrm{~g}$ ). The many observational studies conducted in both well nourished and deprived populations have similarly reported only small associations between energy intake and pregnancy outcome, and the results have not been consistent (e.g. Rush, 1989; Haste et al. 1991; Susser, 1991; Campbell et al. 1996; Godfrey et al. 1996). The majority of dietary studies in this review measured calorific intake, but did not find important relationships with pregnancy outcome. The apparent robustness of pregnancy to variations in energy intake between individuals may in part be explained by the work of Prentice et al. (1989) who have shown that some if not all pregnant women have energy sparing mechanisms which can operate during pregnancy. As Rush (1989) concluded, future research on protein and energy intake could perhaps best be targeted on women most at risk of poor nutrition, in particular those in developing countries.

In industrialized countries, overt undernutrition of macronutrients is uncommon. However, there has been a growing interest in the function of micronutrients. In particular it has been suggested that a substantial proportion of the population - those who smoke - is at risk of poor antioxidant status. Smoking during pregnancy is still widespread: an OPCS survey in the UK in 1990 found that $28 \%$ of pregnant women smoked during pregnancy, and a further $27 \%$ claimed to have given up during pregnancy (Office of Population Censuses and Surveys, 1995). This group is also prone to poor outcomes of pregnancy such as a mean reduction in birth weight of $200 \mathrm{~g}$ and an increased risk of IUGR. Research has shown that smokers generally have a poorer dietary intake of micronutrients than do non-smokers (Margetts \& Jackson, 1993), and even when intakes are equivalent, the concentrations of antioxidant nutrients in the circulation of smokers are lower than in non-smokers. There is a widely supported belief that this is due to the utilization of antioxidants in smoke detoxification reactions. Tobacco smoke contains numerous compounds emitted as gases and condensed tar particles, many of which are oxidants and peroxidants, and are capable of producing free radicals. These can do great damage in the body by enhancing lipid peroxidation and so altering the structure and function of biological membranes. Antioxidant nutrients may defend the body against these attacks: vitamin $\mathrm{E}$ breaks the chain of lipid peroxidation in biological membranes; $\beta$-carotene quenches singlet oxygens, and is more active at lower oxygen tensions than vitamin $\mathrm{E}$; and vitamin $\mathrm{C}$, the only water-soluble antioxidant vitamin, scavenges free radicals in the cytoplasm. The use of micronutrients in this way would mean that less are available to perform other vital functions in the body. This has prompted the USA National Research Council (1980) to set different recommended dietary allowances (RDA) for vitamin C in the two groups $-100 \mathrm{mg} / \mathrm{d}$ for smokers compared with $60 \mathrm{mg} / \mathrm{d}$ for non-smokers.

However, it is also possible that the lower levels of antioxidant nutrients in the blood are a physiological adaptation to oxidative stress from smoking. The so-called antioxidant nutrients can also have pro-oxidant activity under certain conditions: thus rather than combating the oxidative activity of the cigarette smoke, the nutrients could themselves take part in oxidative reactions. The lower antioxidant nutrient levels seen in the plasma of 
smokers may therefore be a strategy to minimize the potential damage which could result from their pro-oxidant activity. This potential for oxidative activity is of particular concern in supplementation trials: unlike in food, where 'antioxidant nutrients' are present as part of a mixture of redox reagents, the nutrients' pro-oxidant activity may predominate when their concentrations are pharmacological.

\section{SCOPE OF REVIEW}

There is now a consensus that in order to reach reliable conclusions reviews must follow the same rigorous scientific principles as original research. Failure to construct a review in a fashion that does justice to the original evidence may result in false or biased conclusions. In particular, articles must be systematically identified and reviewed, and care taken to minimize bias and random error (Gøtzsche, 1987; Mulrow, 1987; Peto, 1987; Oxman \& Guyatt, 1988). Systematic reviews of the literature pertaining to several nutrients already appear in the Oxford Database of Perinatal Trials and 'Effective Care in Pregnancy and Childbirth' (e.g. Mahomed, 1993a,b). However, these publications only consider randomized controlled trials (RCT), and for many antioxidants no RCT exist. Moreover they only investigate adverse outcomes of pregnancy such as preterm delivery and low birth weight rather than effects on mean birth weight and gestation; yet there is growing evidence that small differences in the birth weight of infants considered within the clinically normal range can have profound implications for the infants' future risk of chronic adult diseases such as ischaemic heart disease and diabetes (Barker et al. 1989, 1992, 1993; Hales et al. 1991) which are far more important causes of morbidity and mortality in industrialized countries than are preterm delivery or IUGR. Another systematic review, including observational data, is therefore warranted.

The literature review was limited to original articles which predominantly examined the effect of nutrition on pregnancy outcomes in humans. Studies which focused on medical complications of pregnancy including pregnancy induced hypertension, toxaemia, placenta praevia, abruptio placentae, preterm premature rupture of the membranes, and congenital malformations of the fetus were not included in the search. The view focused on Caucasian women who were not suffering any acute illness. While work dealing with a population of mixed ethnicity was included, that conducted solely with women of other racial origin was not.

\section{IDENTIFICATION OF PAPERS}

A systematic search of MEDLINE, Index Medicus, and other bibliographical sources was conducted to identify relevant studies published in English for the years 1984-November 1995. This was complemented by a 'snowball procedure' whereby the references cited in each article were scrutinized for further research published since 1984.

\section{REVIEW CRITERIA}

Each paper was reviewed against predetermined criteria such as appropriateness of dietary assessment methods and storage conditions for blood samples. A grade was then assigned according to methodological quality. Some studies have been assigned more than one grade, for example where inadequate dietary assessment methods were used but rigorous techniques were employed to determine blood nutrient concentrations. The grades are shown in the text as: (A) high methodological quality; (B) attention paid to good methods, and most criteria met; (C) discarded because of methodological inadequacies. 
An independent causal effect was judged to be demonstrated if the effect size was greater than zero and sampling variation could be excluded as an explanation, i.e. $P \leqslant 0.05$. The size of the effect - for example the difference in means in a matched cohort study, or partial regression coefficients from multiple linear regression analyses - was then assessed and its clinical significance considered. Where no significant effect was reported, the power of the study was assessed.

The literature search identified 33 relevant studies (many of which dealt with more than one nutrient or outcome), of which 32 were located and reviewed. The results for each nutrient are presented separately below. Full details of the methods used to identify and review papers are available on request to the author.

\section{ZINC AND PREGNANCY OUTCOME}

\section{BACKGROUND}

These are several pathways by which a poor intake of zinc or low body zinc status could adversely affect pregnancy outcome. Zinc is a cofactor for a number of important enzymes, in particular DNA polymerase $(E C 2.7 .7 .7)$ : a deficiency of this mineral could therefore impair DNA synthesis, cell division, protein accumulation and fetal growth. Zinc is also involved in the metabolism of oestrogen, progesterone, prostaglandins, carbohydrate and fats; and all nuclear receptors for steroids are zinc finger proteins. Moreover recent reports (Kuhnert et al. 1987a, b, 1992) indicate that zinc is tied up in the placenta of smokers due to cadmium (from cigarette smoke) stimulating the synthesis of metallothionein. The authors go on to suggest that this results in poor fetal growth. Smokers with a poor intake of zinc could therefore be at particular risk of adverse pregnancy outcome.

More work has been published on the effects of zinc status on pregnancy than on any other nutrient with antioxidant activity. This is somewhat surprising given that no biochemical measure of zinc status is universally accepted, with serum zinc in particular being affected by a variety of non-nutritional factors, including circadian rhythms and stress at time of sampling.

\section{RESULTS}

Fourteen studies were located which fulfilled all or most of the methodological criteria (A), 9 fulfilled some criteria (B), and 5 were discarded (C). Many of these investigated more than one outcome of pregnancy, and treating each outcome separately gave $26 \mathrm{~A}$-graded studies, $14 \mathrm{~B}$, and 8 as $\mathrm{C}$. (NB: where associations were found, they were consistent in direction across studies.) Few studies gave details of how the anthropometric measures were taken and of the inter-observer variation. In most cases outcome measures were simply abstracted from the subject's medical case notes. It was difficult to assess the clinical significance of many of the associations reported in the literature because only the results of significance tests were reported.

\section{Birth weight and/or intrauterine growth retardation}

Fourteen (A), 9(B) and 4(C) studies dealing with one or more of these outcomes were located and reviewed.

Zinc supplement trials. Four (A) reports were randomized controlled trials. The best of these, by Mahomed et al. (1989), included 494 randomly selected pregnant women who were then randomly assigned to a placebo or zinc supplement; there were no significant differences in habitual dietary intake of zinc between the two groups. This study failed to show any difference between the groups in birth weight, rates of low birth weight deliveries, 
mean ponderal index, mean head circumference or mean crown--heel length. The analysis was conducted on an 'intention to treat' basis, and when repeated using compliers only, the same results were obtained. The authors acknowledge that different results might have been obtained had the sample been of poorer general nutrition (this sample was predominantly middle class), or if a larger sample had been used (though appropriate sample size calculations had been performed). Another RCT of a similar size was conducted by Cherry et al. (1989). In this study adolescent mothers were randomly allocated to either a zinc supplement or a placebo in addition to a multivitamin and iron tablet which was given to all mothers. The reported compliance was good, and the analysis appears to have been conducted on the groups as randomized (rather than using compliers only). Unfortunately, the method used to collect data on dietary intake was poor. There was no difference between the groups in rate of low birth weight deliveries. Smokers had babies with significantly lower mean birth weights than non-smokers. However, when the analysis was stratified by smoking status (in case of potential confounding from smoking), the placebo and treatment groups did not differ in terms of mean birth weight.

Robertson et al. (1991) in a much smaller study (72 women supplemented with zinc + iron + folate and 62 controls given iron + folate only) showed a non-significant increase in mean birth weight (43.8 g) in the supplemented group - this group had more smokers than the controls, so the opposite result would have been expected if the zinc had no effect. Very low numbers of women agreed to participate in the trial, so it is possible that statistically significant results would be achieved with a larger sample size. However, the low participation rate, and the analysis of data from only those women complying with the treatment, also raise questions about the generalizability of the results. Since no dietary information was collected, it is not possible to determine whether the supplement was effective in this population because there was a lower baseline intake of zinc than in Mahomed's study (Mahomed \& Hytten, 1989), where no effect on birth weight was seen. Simmer et al. (1991), working with women at risk of having a growth retarded baby, showed a significantly lower rate of IUGR in the supplemented group compared with controls $(0$ and 4 cases in the supplement and placebo groups respectively), but found no differences in mean birth weight. Again, no dietary information was collected, and the analysis considered only those women who complied with treatment (only about half of the 56 women randomized). There was also one further paper by Kynast \& Saling (1986) which partly fulfilled the criteria (B), investigating the use of supplements. This study was reasonably large, involving 179 supplemented women, and 345 controls. In this study, as in Simmer's, there were more SGA infants (and also more large-for-gestational-age infants) in the control group, but no differences in mean birth weight between the two groups. However, no placebo was given to the controls, and it was not clear how the supplemented group were chosen: no formal randomization appears to have been carried out, neither do the women comprise a high risk group. Not surprisingly, there were substantial differences between the groups in terms of confounders such as smoking habit.

Conclusions from the supplement trials. The studies reviewed above did not provide good evidence for an increase in birth weight when a zinc supplement was used, or for a reduction in the incidence of low birth weight babies. This is consistent with the findings reported in Kramer's review (1987). There is some evidence to suggest that zinc supplements can reduce the incidence of IUGR in women identified as being at risk of this outcome, but further RCT are needed before conclusions can be drawn with confidence. Mahomed (1993a) reached similar conclusions in an overview of zinc supplementation in pregnancy, which included earlier trials but only investigated low birth weight and preterm delivery as outcomes. 
Two of the studies reviewed here (Cherry et al. 1989; Robertson et al. 1991) used combined supplements of iron and zinc: since iron can affect the absorption of zinc, it is not possible to make straightforward comparisons between these studies and those using zinc alone. Future studies should use zinc-only supplements, and provide details of the amount of elemental zinc available from the supplement to facilitate comparisons between studies.

Cohort studies. There were 4(A) and 3(B) cohort studies which analysed maternal zinc status in relation to mean birth weight or low birth weight. Wells et al. (1987) (A) measured leucocyte zinc - considered by many to be a better measure of body status than serum zinc levels - in a randomly selected cohort of 70 pregnant women. The authors demonstrated significantly lower leucocyte zinc levels in women delivering babies weighing less than the 10th percentile compared with those delivering heavier babies, although no adjustments were made for the parity of the women. They suggested the use of leucocyte zinc levels as a diagnostic tool.

Serum zinc was studied by Neggers $e t$ al. in a cohort of 476 women (364 black, 112 white) of lower socioeconomic status. They published two reports of this work (A, A) (Neggers et al. 1990, 1991). Unlike most earlier studies which used only a single sample of serum zinc, they took samples both early ( 16 weeks) and late (32 weeks) in gestation, so that the analysis could be adjusted for the effects of haemodilution along with other recognized confounders. Zinc concentration at each of these sampling times, and also the rate of fall in zinc concentration during pregnancy, were found to be independent predictors of birth weight (after adjusting for confounders, the correlation coefficient for serum zinc and birth weight $=0.32, P \leqslant 0.001$ ), the difference in birth weight between mothers with serum levels in the lowest and highest quartiles being equivalent to $300 \mathrm{~g}$, a clinically significant difference. The authors also identified a threshold level of $600 \mathrm{mg}$ zinc/ $/ 1$ late in pregnancy, below which the odds ratio (OR) for giving birth to a low birth weight baby rapidly increased from near unity to $5 \cdot 8(95 \%$ confidence interval $(\mathrm{CI})=1 \cdot 8-16 \cdot 4)$.

These two studies contrast with the remaining four studies investigating maternal plasma or serum zine concentrations and fetal growth (Kuhnert et al. 1987 a (B); Bro et al. 1988 (A); Lazebnik et al. 1988 (B); Wasowicz et al. 1993 (B)). They all used maternal venous blood samples taken at or just prior to delivery. No association between maternal plasma zinc concentration and mean birth weight was shown in the Lazebnik and Kuhnert studies, and Wasowicz (whose study design was unclear) was unable to show an association with low birth weight. The Bro paper examined the relationship between maternal serum zinc concentrations and IUGR, but this well conducted study of 500 Danish women could show no association between the variables, and unfortunately no data on mean birth weight were presented. However, all these studies suffered from the use of serum or plasma zinc which is, at best, a marker only for short term zinc status. Therefore the possibility remains that a zinc deficiency earlier in pregnancy caused some insult to growth, but that maternal serum zinc levels equalized, perhaps as a result of the reduced fetal growth, by the time of delivery. The findings of these studies are also unsurprising since the work would be expected to have only sufficient statistical power to detect wide variations in birth weight.

Two cohort studies examined the effects of dietary zinc intake on birth weight. Haste $e t$ al. (1991) (A), in a well conducted if rather small study which examined many nutrients, used 7-d weighed records in randomly selected cohorts of smoking $(n=72)$ and nonsmoking $(n=97)$ pregnant women. They found that zinc intake explained $2 \cdot 4 \%$ of the variance in birth weight after adjustment for confounders $(P \leqslant 0.05)$. Moreover, the amount of variance explained by smoking was reduced by almost the same amount when the nutrient was added to the model, suggesting that some of the reduction in birth weight 
associated with smoking could be mediated by low zinc intakes. The authors also investigated the alteration in zinc intake which occurred between 28 and 36 weeks, and found that a $1 \mathrm{mg}$ decrease in zinc intake was associated with a reduction in birth weight of $49 \mathrm{~g}$ (after adjustment for the effects of smoking, social class and alcohol consumption). However, while of potential clinical importance, this result should be interpreted with care, as response rates had fallen to $69 \%$ for non-smokers and $54 \%$ for smokers by 36 weeks.

The other dietary study by Scholl et al. (1993) fulfilled all the methodological criteria, though only zinc intake was reported and not any other nutrients. This study related to 818 low income pregnant American women. Those with low zinc intake ( $\leqslant 6 \mathrm{mg} / \mathrm{d}$, i.e. $40 \%$ US RDA) were found to have double the risk of giving birth to low birth weight infants $(\mathrm{OR}=2 \cdot 1$; CI 1.19-3.67) compared to those with moderate or high intake (equalling or exceeding the US RDA).

Case-control studies. Four (A) and four (B) case-control studies were located which investigated maternal zinc status. All focused on IUGR, and most examined less than 40 cases. The best marker available for zinc status is leucocyte zinc levels: as discussed earlier this is much less prone to non-nutritional causes of variation, and is a longer term marker of zinc status than are serum or plasma levels. The following studies assessed leucocyte zinc: Simmer \& Thompson in two investigations graded (A) and (B) respectively (Simmer \& Thompson, 1985b; Simmer et al. 1985). Further details of this study were reported in another paper (Simmer \& Thompson, 1985a). This work showed that polymorphonucleocyte (PMN) and mononucleocyte zinc levels were lower in women delivering small for gestational age babies compared to controls. They also reported that PMN zinc was significantly lower in smokers than non-smokers, and suggested that smoking status and/or low PMN zinc levels could identify $85 \%$ of mothers at risk of IUGR. In contrast Jepsen \& Clemmenson (1987) (B) could demonstrate no differences in leucocyte zinc between cases and controls, but took no account of potential confounding factors such as smoking status. It should be noted that these studies used blood samples taken at different times - Simmer 24-48 h post partum, and Jepsen \& Clemmenson either before or after delivery - but since leukocyte zinc is a medium to long term marker the results should not be substantially affected.

Jepsen \& Clemmenson (1987) also provided data on maternal serum zinc levels, but these were discarded because serum zinc concentrations are influenced by the stage of gestation, and no account was taken of this in the study. Two (A) papers did measure plasma or serum zinc levels at standardized stages in gestation among women hospitalized owing to carrying an IUGR baby and controls. Both of these used two blood samples taken during pregnancy (one in the second and one in the third term), and so avoided the difficulties of trying to relate an outcome to a short term marker or nutritional status taken at or after delivery. Tamura et al. (1992) reported finding no differences in serum zinc levels between the groups in their nested case-control study. The categories were then combined for further analysis, but no consistent relationships were found between serum zinc levels and birth weight or incidence of IUGR at either of the sampling times (18 and 30 weeks gestation). In contrast, Borella's study (Borella et al. 1990), with only 16 cases and 35 controls, showed that plasma zinc levels were significantly higher in the IUGR groups in the third (but not the second) trimester.

The lack of association found by Tamura et al. (1992) was also reported by Simmer \& Thompson (1985b) (A). However, this contrasts with their results in the same cohort using leucocytes, and the use of a short term marker on a sample obtained post partum is probably inappropriate. No significant association between maternal serum/plasma zinc and IUGR was reported in either of the B-rated papers (Tuttle et al. 1985; Nasrat et al. 
1992). Nasrat et al. took samples of fetal blood from 11 growth retarded fetuses and 27 controls between 18 and 40 weeks gestation. While plasma zinc levels and the ratio of maternal:fetal plasma zinc were lower in fetuses with symmetrical growth retardation compared with controls of a similar gestational age, these differences were not significant. The other study (Tuttle et al. 1985) compared plasma zinc concentrations and plasma volumes at several stages in pregnancy among a group of 33 women at risk of having a growth retarded baby and 31 apparently unmatched controls. Although there was no difference in mean plasma zinc concentration between the groups at any of the stages of gestation tested, the group at risk of having an IUGR baby had significantly poorer plasma volume expansion, and a lower intravascular mass of zinc late in pregnancy. However, both within and between the groups there was no addition between intravascular zinc mass and the risk of actually delivering an IUGR baby.

The dietary intake of zinc in pregnancy has been studied much less than biochemical status. Tuttle et al. (1985) (A), in addition to their work on plasma zinc discussed above, performed good dietary assessments at 30 and 35 weeks of gestation. While their work showed that the dietary intake of zinc in both groups was about $30 \%$ of the UK RDA, it failed to show any differences in intake between the groups. Two other studies (Simmer \& Thompson, 1985a; Simmer et al. 1987) graded (B,B) measured the dietary intake of randomly selected mothers of IUGR babies. While they found that zinc was the only nutrient to vary significantly between the groups, there was great potential for bias in these studies, as the 7-d diet history used to collect dietary data was administered post partum.

Conclusions from the cohort and case-control studies. There is evidence that low leucocyte or plasma zinc levels are associated with increased rates of low birth weight and lowered mean birth weight. The failure of most RCT to improve birth weight or reduce the incidence of low birth weight might therefore suggest that the low zinc levels are a result rather than a cause of these outcomes. However, good dietary cohort studies have also reported low zinc intake in the second trimester being associated with poor outcome, and it may therefore be that zinc status is positively related to the growth of the fetus, but supplementation of a well nourished population brings no further improvements. RCT which incorporate rigorous assessment of dietary intake are therefore needed before firm conclusions can be made.

The data concerning the association between zinc and IUGR are more equivocal. The balance of evidence currently points to an association between low leucocyte zinc and IUGR, though this finding has not been supported by work on serum or plasma levels, possibly because of the inherent problems of using serum zinc as a biomarker. Leucocyte zinc is a much more reliable measure of body zinc status, but its use in cohort studies is currently hindered by the great cost of the assay: it is difficult to see how observational research into the relationship of maternal zinc status and IUGR can be furthered until technological advances are made to improve the throughput of this assay. There are few data available on the effects of dietary intake on IUGR: a nested case-control study in a large scale dietary assessment programme would provide a good opportunity to clarify the issue.

\section{Preterm delivery and/or gestational age at delivery}

Two RCT fulfilled all the methodological criteria. Mahomed et al. (1989) found no significant differences in mean gestational age at delivery, or in the number of preterm births between women receiving the zinc supplements or placebo. However, as discussed earlier, the population may not have had sufficiently poor dietary intakes to show an effect. Similarly the population rates of preterm delivery may have meant that the study lacked 
statistical power to detect differences in the rate of this outcome. Cherry et al. (1989) in a slightly larger sample comprising pregnant adolescents - a group at risk of nutritional compromise - could find no overall differences between the two groups, but when the sample was subdivided according to weight before and during the pregnancy they found that rates of preterm births were about $10 \%$ lower $(P \leqslant 0.05)$ in normal weight supplemented women compared with normal weight controls. In underweight multiparous women the zinc supplement was associated with an increase of 2.8 weeks in mean gestational age at delivery. However, it is not easy to explain why the supplements were not effective in other subgroups, or why they were effective in multiparous women who were underweight at delivery but not in primiparous women. In addition, the fact that multiple comparisons were used, which do not appear to have been decided upon a priori, means that the results must be reviewed with some caution. One further (B) study also indicated a significant relationship between zinc supplementation and reduced rates of preterm delivery (Kynast \& Saling, 1986), but the work suffered design problems and the data were not analysed on an 'intention to treat' basis.

Only two cohort studies were located which reported the effects of biochemical zinc status on gestational age, both of which reported a positive relationship between serum zinc concentration and the gestational age of the infant at delivery. Neggers et al. (1991) (A) measured maternal zinc concentrations at two stages in pregnancy, and found that at each time point an increase of $10 \mu \mathrm{g} / \mathrm{l}$ of serum zinc was associated with an average increase in gestational length of $1-2 \mathrm{~d}$ (in multiple linear regression, $\beta=0 \cdot 17, P \leqslant 0 \cdot 001$ ). Lazebnik et al. (1988) (B) reported that gestation was $3 \mathrm{~d}$ longer in the women with serum zinc levels above the median. Only one cohort study of dietary intake of zinc investigated gestational age as an outcome (Haste et al. 1991). (A). This measured dietary intake at 28 and 36 weeks and showed no association between the variables.

Preterm delivery was investigated in only two studies (B, B) measuring plasma zinc (Bro et al. 1988; Wasowicz et al. 1993). No association was found between plasma zinc and preterm birth. Bro's cohort study lacked statistical power and the design of Wasowicz's work is unclear (it appears to be an enriched cohort study). Further, the studies used blood samples taken just prior to delivery (rather than at a standardized gestational age): during a normal pregnancy plasma volume expansion results in falling zinc concentrations with increasing gestational age, so even were low serum zinc associated with preterm delivery, the concentrations measured would not differ from those seen in women delivering at term. Hence, the studies would be unlikely to detect a relationship even if one existed.

Perhaps the most interesting data on preterm delivery and zinc come from the only dietary study available (Scholl et al. 1993). While this reported intakes of zinc only, clinically significant associations between intake and preterm delivery were reported in the cohort of 818 women. The risk of preterm delivery was increased when dietary intake was low, particularly when rupture of the membranes preceded labour (OR $=2 \cdot 18(95 \% \mathrm{CI}$ 1.26-3.79) for preterm delivery, and 3.87 (95\% CI 1.85-8.07) for very preterm delivery).

Conclusions. It is important to realize that the factors affecting mean birth weight or gestational age may not be the same as those influencing IUGR, preterm delivery and low birth weight. For example, a factor may affect the middle or upper range of the distribution of birth weights or gestational ages without affecting infants classified as SGA or preterm. Failure to recognize this fact has often led to confusion in the interpretation of apparently conflicting results from different studies. For example, two studies may investigate the effects of a particular factor on gestational duration, but whereas one reports its results in terms of preterm delivery (prior to 37 weeks gestation), the other reports mean gestational age at delivery. 
Blood zinc level appears to have a positive association with mean gestational age, but while the available evidence has shown association between intake late in pregnancy and mean gestational age, the evidence available from RCT suggests that supplementation may be effective only among malnourished women. Further work, preferably an RCT which includes baseline dietary assessment early in pregnancy, is warranted to clarify the situation among women at risk of compromised nutritional status.

Given the clinical significance of preterm delivery, there is a surprising lack of research investigating zinc and the condition. The currently available results from a dietary cohort study and RCT seem to point to a negative relationship between level of zinc intake and incidence of preterm delivery. The different findings relating to zinc for mean gestational age and preterm delivery point to a differing role for zinc in the aetiology of the two outcomes. There is an urgent need for an appropriately sized case-control study, or perhaps better, a cohort study in women at risk of having a second preterm delivery, which measures a long term biomarker of zinc status, e.g. leucocyte zinc, to establish whether there are grounds to mount a large scale randomized controlled trial of zinc supplementation.

\section{SUMMARY OF THE EFFECT OF ZINC ON PREGNANCY OUTCOME}

Reliable information on the association between zinc and pregnancy outcome is somewhat meagre. Largely responsible are the methodological inadequacies of most published work (or failure to report methods in sufficient detail to allow judgments to be drawn). The lack of consensus about an appropriate and reasonably priced biochemical marker adds to the difficulties of interpretation of work on zinc, and suggests that future work should focus on dietary intake or supplements of the nutrient.

At present, the balance of evidence suggests that low serum and leucocyte zinc levels are associated with increased rates of low birth weight and lowered mean birth weight. However, it appears that either the relationship may not be causal or the populations studied to date have been sufficiently well nourished to show no improvement from supplementation regimes. In contrast, there is some evidence that poor zinc status and dietary intake is associated with IUGR, reduced mean gestational age and preterm delivery, and that supplements of zinc could produce clinically relevant improvements in outcome in women at high risk. Large randomized controlled trials of either zinc supplements or dietary intervention, which incorporate rigorous assessments of dietary intake, are now needed to confirm or refute these findings.

\section{VITAMIN C AND PREGNANCY OUTCOME}

\section{BACKGROUND}

Most work on vitamin $\mathrm{C}$ status during pregnancy relates to premature rupture of the membrane on the basis that poor collagen formation and/or maintenance leads to rupture. However, this outcome is not included in the review because the literature is insufficiently clear about hospital policy regarding induction of labour in women with ruptured membranes: in many centres only women refusing induction go on to become true cases of premature rupture of the membranes, as the rest are routinely induced well within $24 \mathrm{~h}$ of rupture. There has been little work on other outcomes, despite the important metabolic functions of the vitamin, including its key role in collagen synthesis and therefore bone formation. Further, ensuring a dietary intake of vitamin $\mathrm{C}$ at each meal is a more effective way to safeguard an adequate uptake of iron in the gut than by increasing iron intake. 
Therefore it would be expected that poor dietary intake of vitamin $\mathrm{C}$ would be associated with the outcomes which result from iron-deficiency anaemia in pregnancy.

\section{RESULTS}

Only five papers were located for this topic, two of which were rated A, one B and one C. One of these, an RCT graded A, investigated the effect of nutritional counselling on the outcome of pregnancy in rural Greece (Kafatos et al. 1989). However, while biochemical measurements were made of vitamin $\mathrm{C}$ and other nutrients, the analysis did not separate out the contribution of individual nutrients to the improved pregnancy outcomes seen with counselling.

\section{Mean birth weight, IUGR and low birth weight}

Only Haste et al. (1991) (A) addressed the effect of dietary vitamin C intake on mean birth weight. This study measured dietary intake of 97 randomly selected non-smokers and 72 heavy smokers at 28 and 32 weeks gestation. While the smokers consumed less vitamin $\mathrm{C}$ than non-smokers, in multiple linear regression with other variables - including smoking, social class and other nutrients - neither intake of the vitamin at 32 weeks nor the change in its intake between 28 and 36 weeks explained a significant amount of the variance in birth weight. The potential relationship between vitamin $\mathrm{C}$ intake and SGA infants was also investigated. Neither intake at 28 or 36 weeks, nor the fall in intake between these times, differed significantly between mothers of small- and adequate-for-gestational-age infants. However, this study lacked the power to detect small differences in intake or outcome. Delivery of SGA infants was also an outcome investigated in Arbuckle's follow-up study of pregnant women who were involved in the Nutrition Canada project (Arbuckle \& Sherman, 1989) (B). This study used serum vitamin C from 57 mothers who subsequently gave birth to SGA babies and 680 controls. The risk of giving birth to a baby which was SGA but not preterm was not significantly associated with serum vitamin $C$, whether or not the analysis was adjusted for other variables.

\section{Preterm delivery and/or gestational age at delivery}

Only Arbuckle's cohort study (Arbuckle \& Sherman, 1989) (B) examined vitamin C status in relation to preterm delivery (all the babies were of adequate size for gestational age). The study compared 68 women who gave birth to preterm infants and 680 controls (data were collected prospectively). They showed that while risk of preterm delivery was significantly associated with serum vitamin $\mathrm{C}$ levels when no other factors were included in the analysis - unadjusted $\mathrm{OR}=0 \cdot 48 ; \mathrm{CI}=0 \cdot 25-0.90$ - in multiple logistic regression only maternal education, household income and sex of the infant were significant. Unfortunately the authors did not present any results which considered pregnant smokers and nonsmokers separately. Mean gestational age at delivery and dietary intake of vitamin $\mathrm{C}$ were investigated by Haste et al. (1991) (A). Neither dietary intake of the vitamin at 32 weeks gestation nor change in intake over the third term of pregnancy were significant predictors of mean gestational age, though the earlier comments about this study also apply here.

As in the case of zinc, the most appropriate biochemical marker of vitamin $C$ status is not clear. Serum and plasma vitamin $\mathbf{C}$ concentrations are commonly employed but strongly reflect recent dietary intake and therefore give an index of only short to medium term body status. Leucocyte vitamin $\mathrm{C}$ concentration is considered a good index of long term status. However, use of the entire 'buffy coat' compartment may lead to serious errors since different cell types vary in their concentrations of vitamin $\mathrm{C}$, and under certain conditions one cell type may proliferate much more than others. It is therefore preferable 
to use only one fraction of white blood cells, but the cost of the necessary separation procedures is currently prohibitive.

\section{SUMMARY}

No studies have shown an association between the amount of vitamin $\mathrm{C}$ consumed, or serum levels of the vitamin, and the outcome of pregnancy. However, most of these studies have had insufficient power to detect small differences between groups. The rapid decay of vitamin $\mathrm{C}$ in blood samples complicates the interpretation of biochemical studies: unless highly standardized techniques are used, the decay of samples prior to storage may completely obscure interindividual differences in concentration of this nutrient. A further consideration concerns the use of regression analyses. Vitamin $\mathrm{C}$ intake and serum levels are both known to be highly correlated with smoking (and also other factors such as household income). Therefore, inclusion of these factors in a multiple regression analysis is likely to mask the effects of vitamin $\mathrm{C}$, even if the vitamin is crucial to the aetiology of the condition studied. The only way to separate the effects of, say, smoking and vitamin $\mathrm{C}$ on birth weight in a cohort study, to determine which is the true predictor and which a confounder, would be to look for an association between levels of the vitamin and birth weight within a cohort of smokers. This would then demonstrate whether vitamin $\mathrm{C}$ is a confounder of smoking, with smoking being the important variable; or whether vitamin $\mathrm{C}$ is an important factor in its own right, but whose effect would usually be overlooked through its high correlation with smoking. The present situation is therefore the same as that reported by Kramer in 1987: no conclusions can be drawn from presently available studies, and more of higher methodological quality are required.

\section{CAROTENOIDS AND PREGNANCY OUTCOME}

\section{BACKGROUND}

Apparently no mechanism has been proposed to explain any effect of carotenoids on fetal growth. However, their strong antioxidant activity may be important. Fibrinogen synthesis is stimulated by endothelial damage, most of which results from the activity of oxidants (Gey, 1990). Therefore low carotenoid status may result in high fibrinogen concentration in the blood during pregnancy, which in turn could limit placental perfusion through its effects on blood viscosity, so compromising fetal growth. (This mechanism may, of course, apply to any of the other antioxidants reviewed here.) There has also been preliminary research which suggests that carotenoids have a growth stimulating action at the cellular level similar to that of retinol. Finally, any effect of carotenoids on fetal growth may occur via their influence on serum retinol concentrations.

\section{RESULTS}

Only two references were located which investigated the effects of carotenoids in pregnancy, one of which was graded A for methodology, and one B. Both were cohort studies and investigated mean birth weight or rate of low birth weight.

Metcoff et al. (1989) studied serum $\beta$-carotene levels in 214 non-smokers and 196 smokers, collecting a second blood sample at 36 weeks gestation from $77 \%$ of the women tested at 19 weeks. This publication was graded B as, despite fulfilling all the other methodological criteria, no storage methods for blood samples were given, either in this or previous papers from the study. A clear interaction between smoking and carotene levels was shown (smokers with high carotene levels had babies with higher birth weights than 
smokers with low carotene in mid-pregnancy: the partial regression coefficient $=8 \cdot 14$, $\mathrm{SE}=2 \cdot 87, P=0.005$, which predicts a difference in birth weight of $323 \mathrm{~g}$ between those smokers with carotene levels equalling the group mean and those with levels $1 \mathrm{SD}$ below the mean). The change in carotene levels between 19 and 36 weeks was also significantly related to birth weight, though the size of the association was less. This study showed that women who smoked and had infants weighing under $3000 \mathrm{~g}$ had the greatest decrease in serum $\beta$-carotene during pregnancy. In contrast, mothers whose carotene levels increased, regardless of their smoking habit, had babies of over $3600 \mathrm{~g}$. Further, the authors had previously shown that maternal cholesterol levels correlated with birth weight: the present work showed that in smokers this relationship was dependent on the level of serum $\beta$-carotene simultaneously observed.

The other study investigated dietary intake of carotene. Haste et al. (1991) showed that intakes of carotene were significantly lower in smokers than non-smokers. Carotene intake at 36 weeks gestation explained $1.9 \%(P \leqslant 0.06)$ of the variation in birth weight in multiple linear regression with other factors including smoking and different nutrients. The variance explained by smoking was reduced by an approximately equivalent amount when carotene was added to the model, suggesting that some of the effects of smoking on birth weight can be explained in terms of different carotene intakes. Gestational age at delivery was not associated with dietary carotene consumption.

\section{SUMMARY}

While the available data are very limited, they do suggest that in smokers serum carotene may have an important association with birth weight. There are many good studies in the general population showing that smokers have lower carotene levels than non-smokers. Therefore, if confirmed, the effect in pregnancy could have important clinical implications. Haste et al. (1991) showed that dietary intake of carotene was lower in smokers than nonsmokers, and that this explained a significant proportion of the variance in birth weight. The fact that the amount of variance explained by smoking decreased by a proportional amount when dietary carotene intake was added to the model supports Metcoff's conclusion that carotene status is important in determining birth weight in smokers. Knowledge about the possible role of carotenoids has therefore improved since Kramer's (1987) review, but a large amount of work is still required. The most appropriate way to investigate the effects of carotenoids further would be to study carotene levels and birth weight in a large cohort of smokers followed, if positive results are obtained, by an intervention trial. Further work is also warranted to investigate the role of the different carotenoid fractions on pregnancy outcome.

\section{VITAMIN E AND PREGNANCY OUTCOME}

\section{BACKGROUND}

As with carotene, no mechanisms by which vitamin $E$ levels could affect fetal growth or length of gestation outcome are immediately obvious, although the general mechanism proposed for antioxidants may apply. An indirect effect via other metabolic pathways, including its influence on red blood cell formation, is also possible.

\section{RESULTS}

Only two papers were located. Dison et al. (1993) (A) investigated the relationship of both maternal serum $\alpha$-tocopherol and the ratio tocopherol:cholesterol to birth weight and gestational age. No correlations were found between the nutrient and these outcomes, but 
the study was small and unfortunately collected maternal samples only $2-3 \mathrm{~d}$ post partum. The other study, by Haste et al. (1991), investigated the effect of dietary intake of vitamin $\mathrm{E}$ on birth weight. While there were significant differences in dietary intake between smokers and non-smokers, and intake fell between 28 and 36 weeks gestation, neither absolute intake nor change in dietary intake had any significant effect on birth weight in multiple linear regression. However, the comments made above about this study also apply to the work on vitamin $\mathrm{E}$ : intake of this nutrient is, in the general population at least, highly correlated with smoking habit. It is therefore not surprising that the nutrient does not explain significant amounts of the variation in birth weight when added to a model containing smoking as a factor. A cohort study investigating the dietary intake and pregnancy outcome of pregnant smokers would be able to demonstrate whether vitamin $\mathrm{E}$ is important to birth weight in this group.

\section{SUMMARY}

This review agrees with the earlier one by Kramer (1987): there is insufficient work of good methodological quality to allow any conclusions to be drawn about the effect of this nutrient in pregnancy.

\section{IRON AND PREGNANCY OUTCOME}

\section{BACK GROUND}

Maternal erythropoiesis rises throughout pregnancy, but there are even greater increases in plasma volume. This results in progressively falling haemoglobin concentrations and haematocrit levels. Until recently, iron was routinely given to pregnant mothers on the assumption that returning haematological profiles toward those seen in the non-pregnant state would lower the incidence of anaemia, so improving pregnancy outcome. It is true that anaemia could lead to impaired oxygen delivery to the fetus, and so interfere with its growth, and that iron deficiency, whether associated with anaemia or not, could adversely affect fetal growth owing to its key role in several enzymes, including cytochrome oxidase (EC 1.9.3.1). However, the haematological profiles seen in pregnant women usually represent a normal physiological adaptation to pregnancy rather than being pathological.

The view that iron supplements should be given routinely has been challenged by a number of randomized controlled trials of routine iron (or iron + folate) supplementation $v$. supplementation only when indicated on haematological grounds. The results of these RCT have been summarized in overviews by Mahomed (Mahomed \& Hytten, 1989; Mahomed 1993b). The results of the trials which met his inclusion criteria were consistent, and were combined in a meta analysis. No detectable effects on low birth weight or preterm delivery were seen with routine supplementation. These overviews did not include data on the effect of iron on mean birth weight or gestational age, or the influence of high haematological parameters such as high haematocrits on pregnancy, so these will be considered here.

\section{RESULTS}

Only one RCT was identified which investigated the effects of iron supplementation on mean birth weight and mean gestational age. Hemminki \& Rimpela (1991) (A) randomly assigned 2912 women to either routine supplementation with iron or to pragmatic treatment, supplementing only if their haemoglobin levels were fourid to be low on two consecutive visits. Supplementation was stopped in this latter group once haemoglobin (or 
packed cell volume) measurements rose to a predefined level. Mean gestational age seemed shorter in the infants of the non-routinely supplemented group, but only reached statistical significance among boys (where it was 39.9 weeks in the non-routinely and 40.1 weeks in the routinely supplemented groups). As would be expected with a longer gestation, the boys in the routinely supplemented group were also of greater length. No differences were seen between the groups in terms of mean birth weight or placental weight.

Only one cohort study set out to investigate specifically the effects of iron deficiency anaemia in pregnancy, detected by low serum ferritin in addition to low haemoglobin, as distinct from other low haematological parameters e.g. low mean cell volume (MCV) (Scholl et al. 1992) (A). In their cohort of 826 women, the odds of preterm birth more than doubled with iron deficiency anaemia, whereas they were not increased with anaemia from other causes. The group also found that the odds of low birth weight tripled with iron deficiency, but there was no effect on IUGR. Since most of the women diagnosed as anaemic by the usual standards were given iron supplements soon after diagnosis, it can be concluded that any alteration in pregnancy outcome due to iron deficiency must be determined early in pregnancy. Unfortunately, the dietary data collected in this study were poor, so must be discarded.

The remaining three cohort studies (all A-rated), perhaps surprisingly demonstrated that high values for other haematological parameters (e.g. high haematocrits or haemoglobin concentrations) were associated with adverse effects on the fetus. Godfrey et al. (1991) (A), following work suggesting that a high ratio of placental:fetal weight was associated with cardiovascular disease in adults, investigated the associations between haematological measurements, mean birth weight and placental size. This study used retrospective analysis of computerized records from 8684 pregnant women. Both low maternal haemoglobin and the fall in MCV during pregnancy were associated with large placental weight, with the largest placentas occurring in women with low haemoglobin and a large fall in MCV. Birth weight was affected similarly, increasing the lower the maternal haemoglobin and the larger the fall in MCV. But these differences in birth weight between women with low and high haemoglobin levels (or large and small falls in MCV) were proportionally smaller than for placental weight. Only data on the change in the ratio of birth:placental weight were actually presented in the paper, with the highest ratio of placental:birth weight occurring in the most anaemic women with the largest MCV fall (ratio $=0.192$ compared with 0.181 for the least anaemic women with the smallest drop in MCV). The authors acknowledged that maternal smoking reduced placental weight, and also increased the ratio of placental:fetal weight (by lowering birth weight proportionally more). However, they did not adjust for the fact that smokers tend to have raised haemoglobin levels (Nordenberg et al. 1990) while still having a high ratio of placental:fetal weight. Therefore, the true relationship between haemoglobin and placental: fetal weight may have been even stronger than that reported in their paper.

Consistent results were obtained by Knottnerus et al. (1990) (A). They found in their study of 796 pregnant women that low birth weight and preterm birth were significantly more frequent in women with high erythrocyte count, haemoglobin, or haematocrit in the third trimester. For $\mathrm{Hb} \geqslant 8.0 \mathrm{mmol} / \mathrm{l}$, OR for low birth weight $(\mathrm{LBW})=4 \cdot 2$, (CI 1.9-9.1), and for preterm delivery $\mathrm{OR}=2.4$ (CI 1-5.6). For haematocrit $\geqslant 38 \%$, OR for $\mathrm{LBW}=3.0(\mathrm{CI} 1 \cdot 5-6 \cdot 3)$, and $\mathrm{OR}=2.6(\mathrm{CI} 1 \cdot 2-5.9)$ for preterm delivery. The effects of haematocrit (but not the other haematological indices) were still evident when important variables such as smoking, hypertension, and parity were included in multiple linear regression. With a haematocrit of $40 \%$, the OR of preterm delivery or LBW were equivalent to that for smoking eight cigarettes/d. Further, the effects of high haematocrit were independent of smoking, giving pregnant smokers with high haematocrit an even 
poorer prognosis. Findings in the same order of magnitude were obtained by $\mathrm{Lu}$ et al. (1991). They performed a retrospective analysis of the computerized medical records of 17149 American women, all of whom were offered iron and folate supplementation as part of their routine antenatal care. High haematocrits (over $40 \%$ ), whether seen early or late in pregnancy, were associated with preterm delivery and also IUGR in multivariate analysis. The strongest relationship was for haematocrits at or above $43 \%$ at $31-34$ weeks gestation, where the adjusted OR for preterm delivery was $2.5(\mathrm{CI}=1 \cdot 3-3)$. In contrast, no ill effects of low haematocrits early in pregnancy (below 37\%) were seen. A negative association between haematocrit and birth weight, length and placental size was also reported by Hemminki \& Rimpela (1991), who conducted the RCT discussed earlier. This relationship was seen in both the routinely and non-routinely supplemented groups, and they therefore suggest that the correlations reported between high packed cell volume and poor fetal growth are mediated by some mechanism other than iron supplementation.

The effects of dietary intake of iron (and other nutrients) on pregnancy outcome were investigated by Haste et al. (1991), who showed that dietary intake of iron was significantly lower in smokers than non-smokers at 28 weeks of pregnancy, but not at 36 weeks. But in neither group did iron intake explain a significant amount of the variance in birth weight in multiple regression analyses. However, it was unclear whether the use of iron supplements was included in the assessment of dietary intake, or what the policy of supplementation was at the hospital where the study was carried out. It is therefore difficult to draw conclusions about the effect of dietary intake of iron from this study.

\section{SUMMARY}

One good cohort study has indicated that clinically important risks of low birth weight and preterm delivery are associated with iron deficiency anaemia, as opposed to anaemia from other causes which was associated with no increased risk, and that deficiency affects outcome early in pregnancy. However, the studies reviewed here also indicate that, at the other end of the scale, high haematocrit and/or haemoglobin levels are associated with poor outcomes of pregnancy: preterm delivery, LBW and IUGR. The reasons for this association may be complex. If a fetus is growing poorly, the endocrine response of the mother will be impaired and the maternal adaptations, which include increased plasma volume, diminished. Thus high haematocrit would be a response to poor fetal growth. A contrasting scenario would be where the fetus is growing normally, but some vasoconstrictive process contracts plasma volume late in pregnancy. Then the resulting high haematocrit would further compromise uteroplacental circulation by increasing blood viscosity, and so would directly contribute to poor fetal outcome. The randomized controlled trials reviewed by Mahomed have indicated no benefit from routine iron supplementation in terms of preterm delivery, LBW or IUGR, and the studies reviewed here also showed no evidence of benefit to mean birth weight or gestational age. It would seem sensible to avoid prescribing iron supplements to women with high haematocrit values, even though it is not certain that there is a direct relationship between supplementation and high haematocrit.

While high haematological values may have adverse consequences for the immediate health of the infant, it would be unwise to recommend measures to reduce them to 'average' values at present, since the long term health of the infant may then be compromised. There is evidence to suggest that low haematological parameters are associated with a high placental: birth weight ratio, and the work of Barker et al. indicates that a high ratio is strongly associated with cardiovascular disease in later life. Further assessment of the effect of iron status on placental as well as fetal growth is urgently needed. 
In particular, more research is required to determine the effects of dietary intake of iron on pregnancy outcomes both in populations where iron supplements are routine and where they are only given on haematological grounds.

\section{SELENIUM AND PREGNANCY OUTCOME}

\section{BACKGROUND}

Selenium is an integral part of many metalloenzymes, for example glutathione peroxidase $(E C 1.11 .1 .9)$ and formate dehydrogenase $(E C 1.2 .1 .2)$; situated at the active site of these enzymes, it actually participates in the catalytic process. It is therefore essential to the protection of the cell membranes against oxidative damage. Both selenium and vitamin $\mathrm{E}$ deficiency have been associated with the increased haemolysis seen in preterm infants exposed to oxidative stress, and with the increased risk of pulmonary damage in preterm infants treated with oxygen for respiratory distress syndrome. A deficiency of the nutrient in pregnancy would also be expected to have other consequences for the growing fetus, and many areas have low selenium levels in the soil, resulting in low dietary inta kes (National Research Council, 1980). Yet there has been little research on the role o this nutrient except that relating to selenium poisoning.

\section{RESULTS}

Two studies were identified which investigated maternal plasma or serum selenium concentrations. Dison et al. (1993) (A) in their cohort of 58 women found no correlation between maternal plasma selenium concentration 2-3 d after delivery and either the mean birth weight of the infant or its gestational age. This finding is consistent with another well conducted study by Bro et al. (1988) (A) on 500 Danish women which showed no association between maternal selenium (serum samples taken during delivery) and preterm delivery or IUGR. Unfortunately this group did not investigate the relationship of maternal serum selenium to mean birth weight or gestational age. The other study by Wasowicz et al. (1993) (rated (B) as the design was unclear) also found no association between maternal selenium and these outcomes.

\section{CONCLUSIONS}

The available research does not conclusively rule out a role for selenium deficiency in the aetiology of low birth weight or preterm delivery as the studies may have lacked statistical power. Further, they collected serum samples around delivery and, as with zinc, serum selenium is best regarded as a short term measure of body status. It is therefore possible that deficiencies of selenium were present earlier in gestation and affected growth or predisposed preterm delivery, but maternal levels returned to normal by the time of delivery, perhaps even as a result of the compromised fetal development.

\section{OVERALL CONCLUSIONS FROM THE REVIEW}

With the possible exceptions of zinc and routine iron supplementation, the present state of knowledge about associations between micronutrient status and pregnancy outcome is very poor. The majority of studies suffered methodological weaknesses, and reported results only in terms of statistical significance, making it difficult to assess the relative importance of each factor. In most cases there are insufficient grounds to justify randomized controlled 
trials, particularly following the experience with protein supplements where pregnancy was actually adversely affected. Instead large cohort studies are needed, particularly in women at risk of poor pregnancy outcome, or compromised nutritional status, to identify the nutrients which seem to be of most importance.

\section{REFERENCES}

Antonov, A. N. (1947). Children born during the siege of Leningrad in 1942. Journal of Pediatrics 30, $250-259$.

Arbuckle, T. E. \& Sherman, G. J. (1989). Comparison of the risk factors for pre-term delivery and intrauterine growth retardation. Paediatric and Perinatal Epidemiology 3, 115-129.

Barker, D. J. P., Godfrey, K. M., Osmond, C. \& Bull, A. (1992). The relation of fetal length, ponderal index and head circumference to blood pressure and the risk of hypertension in adult life. Paediatric and Perinatal Epidemiology 6, 35- 44.

Barker, D. J. P., Hales, C. N., Fall, C. H. D., Osmond, C., Phipps, K. \& Clark, P. M. S. (1993). Type 2 (noninsulin-dependent) diabetes mellitus, hypertension and hyperlipidaemia (syndrome $\mathrm{X}$ ) : relation to reduced fetal growth. Diabetologia 36, 62-67.

Barker, D. J. P., Winter, P. D., Osmond, C., Margetts, B. \& Simmonds, S. J. (1989). Weight in infancy and death from ischaemic heart disease. Lancet ii, 577-580.

Borella, P. Szilagyi, A., Than, G., Csaba, I., Giardino, A. \& Facchinetti, F. (1990). Maternal plasma concentration of magnesium, calcium, zinc and copper in normal and pathological pregnancies. Science of the Total Environment 99, 67-76.

Bro, S., Berendtsen, H., Nørgaard, J., Høst, A. \& Jørgensen, P. J. (1988). Serum zinc and copper concentrations in maternal and umbilical cord blood. Relation to course and outcome of pregnancy. Sandinavian Journal of Clinical and Laboratory Investigation 48, 805-811

Campbell, D. M., Hall, M. H., Barker, D. J. P., Cross, J., Sheill, A. W. \& Godfrey, K. M. (1996). Diet in pregnancy and the offspring's blood pressure 40 years later. British Journal of Obstetrics and Gynaecology 103, $273-280$.

Cherry, F. F., Sandstead, H. H., Rojas, P., Johnson, L. K., Batson, H. K. \& Wang, X. B. (1989). Adolescent pregnancy: associations among body weight, zinc nutriture, and pregnancy outcome. American Journal of Clinical Nutrition 50, 945-954.

Dison, P. J., Lockitch, G., Halstead, A. C., Pendray, M. R., Macnab, A. \& Wittmann, B. K. (1993). Influence of maternal factors on cord and neonatal plasma micronutrient levels. American Journal of Perinatology 10, 30-35.

Gey, K. F. (1990). On the antioxidant hypothesis with regard to arteriosclerosis. In Scientific Evidence for Dietary Targets in Europe [J. C. Somogyi, editor]. Bibliotheca 'Nutritio et Dieta', vol. 37. Basel: Karger.

Godfrey, K., Robinson, S., Barker, D. J. P., Osmond, C. \& Cox, V. (1996). Maternal nutrition in early and late pregnancy in relation to placental and fetal growth. British Medical Journal 312, 410-414.

Godfrey, K. M., Redman, C. W. G., Barker, D. J. P. \& Osmond, C. (1991). The effect of maternal anaemia and iron deficiency on the ratio of fetal weight to placental weight. British Journal of Obstetrics and Gynaecology 98, 886-891.

Gøtzsche, P. C. (1987). Reference bias in reports of drug trials. British Medical Journal 295, 654-656.

Hales, C. N., Barker, D. J. P., Clark, P. M. S., Cox, L. J., Fall, C., Osmond, C. \& Winter, P. D. (1991). Fetal and infant growth and impaired glucose tolerance at age 64. British Medical Journal 303, 1019-1022.

Haste, F. M., Brooke, O. G., Anderson, H. R. \& Bland, J. M. (1991). The effect of nutritional intake on outcome of pregnancy in smokers and non-smokers. British Journal of Nutrition 65, 347-354.

Hemminki, E. \& Rimpela, U. (1991). Iron supplementation, maternal packed cell volume, and fetal growth. Archives of Disease in Childhood 66, 422-425.

Hurley, L. S. \& Tao, S. (1972). Alleviation of teratogenic effects of zinc deficiency by simultaneous lack of calcium. American Journal of Physiology 222, 322-325.

Jepsen, L. Voss \& Clemmenson, K. (1987). Zinc in Danish women during late normal pregnancy and pregnancies with intra-uterine growth retardation. Acta Obstetricia et Gynecologica Scandinavica 66, 401-405.

Kafatos, A. G., Vlachonikolis, I. G. \& Codrington, C. A. (1989). Nutrition during pregnancy: the effects of an educational intervention program in Greece. American Journal of Clinical Nutrition 50, 970-979.

Knottnerus, J. A., Delgado, L. R., Knipschild, P. G., Essed, G. G. M. \& Smits, F. (1990). Haematologic parameters and pregnancy outcome. A prospective cohort study in the third trimester. Journal of Clinical Epidemiology 43, 461-466.

Kramer, M. S. (1987). Determinants of low birth weight: methodological assessment and meta-analysis. Bulletin of the World Health Organization 65, 663-737.

Kuhnert, B. R., Kuhnert, P. M., Debanne, S. \& Williams, T. G. (1987a). The relationship between cadmium, zinc, and birth weight in pregnant women who smoke. American Journal of Obstetrics and Gynecology 157, 1247 - 1251 .

Kuhnert, B. R., Kuhnert, P. M., Groh-Wargo, S. L., Webster, S., Erhard, P. \& Lazebnik, N. (1992). Smoking alters the relationship between maternal zinc intake and biochemical indices of fetal zinc status. American Journal of Clinical Nutrition 55, 981-984. 
Kuhnert, P. M., Kuhnert, B. R., Erhard, P., Brashear, W. T., Groh-Wargo, S. L. \& Webster, S. (1987b). The effect of smoking on placental and fetal zinc status. American Journal of Obstetrics and Gynecology 157, 1241-1246.

Kynast, G. \& Saling, E. (1986). Effect of oral zinc application during pregnancy. Gynecologic and Obstetric Investigation 21, 117-123.

Lazebnik, N., Kuhnert, B. R., Kuhnert, P. M. \& Thompson, K. L. (1988). Zinc status, pregnancy complications, and labor abnormalities. American Journal of Obstetrics and Gynecology 158, 161-166.

Lu, Z. M., Goldenberg, R. L., Cliver, S. P., Cutter, G. \& Blankson, M. (1991). The relationship between maternal hematocrit and pregnancy outcome [see comments]. Obstetrics and Gynecology 77, 190-194.

Mahomed, K. (1993a). Routine zinc supplementation in pregnancy. Review no. 06944, 28 April 1993. In Pregnancy and Childbirth Module [M. W. Enkin, M. J. N. Kierse, M. J. Renfrew and J. P. Neilson, editors]. (Cochrane Database of Systematic Reviews). Oxford: Cochrane Updates on Disk, Update Software.

Mahomed, K. $(1993 b)$. Routine folate supplementation in pregnancy. Review no. 03158, 28 April 1993. In Pregnancy and Childbirth Module [M. W. Enkin, M. J. N. Kierse, M. J. Renfrew and J. P. Neilson, editors]. (Cochrane Database of Systematic Reviews). Oxford: Cochrane Updates on Disk, Update Software.

Mahomed, K. (1993c). Routine iron supplementation in pregnancy. Review no. 03157, 28 April 1993. In Pregnancy and Childbirth Module [M. W. Enkin, M. J. N. Kierse, M. J. Renfrew and J. P. Neilson, editors]. (Cochrane Database of Systematic Reviews). Oxford: Cochrane Updates on Disk, Update Software.

Mahomed, K. \& Hytten, F. (1989). Iron and folate supplementation in pregnancy. In Effective Care in Pregnancy and Childbirth, pp. 301-317 [1. Chalmers, M. Enkin and M. J. N. C. Kierse, editors]. Oxford: Oxford University Press.

Mahomed, K., James, D. K., Golding, J. \& McCabe, R. (1989). Zinc supplementation during pregnancy: a double blind randomised controlled trial. British medical Journal 299, 826-830.

Margetts, B. M. \& Jackson, A. A. (1993). Interactions between people's diet and their smoking habits: the dietary and nutritional survey of British adults. British Medical Journal 307, 1381-1384.

Metcoff, J., Costiloe, P., Crosby, W. M., Sandstead, H. H. \& Milne, D. (1989). Smoking in pregnancy: relation of birth weight to maternal plasma carotene and cholesterol levels. Obstetrics and Gynecology 74, 302-309.

MRC Vitamin Study Research Group (1991). Prevention of neural tube defects: results of the Medical Research Council Vitamin Study. Lancet 338, 131-137.

Mulrow, C. D. (1987). The medical review article: state of the science. Annals of Internal Medicine 106, $485-488$.

Nasrat, H., Bloxam, D., Nicolini, U., Williams, N., Tannirandorn, Y., Nicolaides, P. \& Roedeck, C. H. (1992). Midpregnancy plasma zinc in normal and growth retarded fetuses - a preliminary study. British Journal of Obstetrics and Gynaecology 99, 646-650.

National Research Council (1980). Recommended Dietary Allowances, 9th ed. Washington, DC: National Academy of Sciences Press.

Neggers, Y. H., Cutter, G. R., Acton, R. T., Alvarez, J. O., Bonner, J. L., Goldenberg, R. L., Go, R. C. P. \& Roseman, J. M. (1990). A positive association between maternal serum zinc concentration and birth weight. American Journal of Clinical Nutrition 51, 678-684.

Neggers, Y. H., Cutter, G. R., Alvarez, J. O., Goldenberg, R. L., Acton, R., Go, R. C. P. \& Roseman, J. M. (1991). The relationship between maternal serum zinc levels during pregnancy and birthweight. Early Human Development 25, 75-85.

Nordenberg, D., Yip, R. \& Binkin, N. J. (1990). The effect of cigarette smoking on hemoglobin levels and anemia screening. Journal of the American Medical Association 264, 1556-1559.

Office of Population Censuses and Surveys. (1995). Infant Feeding 1990: a Survey. London: OPCS.

Oxman, A. D. \& Guyatt, G. H. (1988). Guidelines for reading literature reviews. Canadian Medical Association Journal 138, 697-703.

Peto, R. (1987). Why do we need systematic overviews of randomized trials? Statistics in Medicine 6, $233-240$.

Prentice, A. M., Goldberg, G. R., Davies, H. L., Murgatroyd, P. R. \& Scott, W. (1989). Energy-sparing adaptations in human pregnancy assessed by whole-body calorimetry. British Journal of Nutrition 62, 5-22.

Robertson, J. S., Heywood, B. \& Atkinson, S. M. (1991). Zinc supplementation during pregnancy. Journal of Public Health Medicine 13, 227-229.

Rush, D. (1989). Effect of changes in protein and calorie intake during pregnancy on the growth of the human fetus. In Effective Care in Pregnancy and Childbirth, pp. 255-280 [I. Chalmers, M. Enkin and M. J. N. C. Kierse, editors]. Oxford: Oxford University Press.

Scholl, T. O., Hediger, M. L., Fischer, R. L. \& Shearer, J. W. (1992). Anemia vs iron deficiency: increased risk of preterm delivery in a prospective study. American Journal of Clinical Nutrition 55, 985-988.

Scholl, T. O., Hediger, M. L., Schall, J. I., Fischer, R. L. \& Khoo, C. S. (1993). Low zinc intake during pregnancy: its association with preterm and very preterm delivery. American Journal of Epidemiology 137, 1115-1124.

Simmer, K., Iles, C. A., Slavin, B., Keeling, P. W. N. \& Thompson, R. P. H. (1987). Maternal nutrition and intrauterine growth retardation. Human Nutrition: Clinical Nutrition 41C, 193-197.

Simmer, K., Lort-Phillips, L., James, C. \& Thompson, R. P. H. (1991). A double-blind trial of zinc supplementation in pregnancy. European Journal of Clinical Nutrition 45, 139-144.

Simmer, K., Punchard, N. A., Murphy, G. \& Thompson, R. P. H. (1985). Prostaglandin production and zinc depletion in human pregnancy. Pediatric Research 19, 697-700. 
Simmer, K. \& Thompson, R. P. H. (1985a). Zinc in the fetus and newborn. Acta Paediatrica Scandinavica Supplement $319,158-163$.

Simmer, K. \& Thompson, R. P. H. (1985b). Maternal zinc and intrauterine growth retardation. Clinical Science 68, 395-399.

Stein, Z., Susser, M., Saenger, G., Marolla, F. (1975). Famine and Human Development: The Dutch Hunger Winter of 1944/1945. New York: Oxford University Press.

Susser, M. (1991). Maternal weight gain, infant birth weight, and diet: causal sequences. American Journal of Clinical Nutrition 53, $1384-1396$.

Tamura, T., Goldenberg, R. L., Freeberg, L. E., Cliver, S. P., Cutter, G. R. \& Hoffman, H. J. (1992). Maternal serum folate and zinc concentrations and their relationships to pregnancy outcome. American Journal of Clinical Nutrition 56, 365-370.

Tuttle, S., Aggett, P. J., Campbell, D. \& MacGillivray, I. (1985). Zinc and copper nutrition in human pregnancy: a longitudinal study in normal primigravidae and in primigravidae at risk of delivering a growth retarded baby. American Journal of Clinical Nutrition 41, 1032-1041.

Wasowicz, W., Wolkanin, P., Bednarski, M., Gromadzinska, J., Sklodowska, M. \& Grzybowska, K. (1993). Plasma trace element $(\mathrm{Se}, \mathrm{Zn}, \mathrm{Cu})$ concentrations in maternal and umbilical cord blood in Poland: relation with birth weight. Biological Trace Element Research 38, 205-215.

Wells, J. L., James, D. K., Luxton, R. \& Pennock, C. A. (1987). Maternal leucocyte zinc deficiency at start of third trimester as a predictor of fetal growth retardation. British Medical Journal 294, 1054-1056. 